\title{
A Crack between Orthotropic Materials with a Shear Yield Zone at the Crack Tip
}

\author{
V. Loboda $\mathbb{D}$, I. Gergel, T. Khodanen, and O. Mykhail \\ Department of Theoretical and Computational Mechanics, Oles Honchar Dnipro National University, \\ Gagarin Avenue 72, Dnipro 49010, Ukraine \\ Correspondence should be addressed to V. Loboda; loboda@dnu.dp.ua
}

Received 25 December 2018; Revised 1 May 2019; Accepted 22 May 2019; Published 25 June 2019

Academic Editor: Ricardo Branco

Copyright (C) 2019 V. Loboda et al. This is an open access article distributed under the Creative Commons Attribution License, which permits unrestricted use, distribution, and reproduction in any medium, provided the original work is properly cited.

A plane problem for a crack between two anisotropic semi-infinite spaces under remote tensile-shear loading is considered. In the framework of the assumption that the crack faces are free of stresses an exact analytical solution of the problem is given on basis of the complex potentials approach. This solution possesses oscillating square root singularities in stresses and in the derivatives of the displacement jumps at the crack tips. To remove these singularities a new model founded on the introduction of the shear yield zones at the crack tips is suggested. This model is appropriate for the cases where interface adhesive layer is softer than the surrounding matrixes. Under this assumption the problem is reduced to the nonhomogeneous combined Dirichlet-Riemann boundary value problem with the conditions at infinity. An exact analytical solution of this problem is presented for the case of a single yield zone. The length of this zone is found from the finiteness of the shear stress at the end point of the zone. Due to such simulation the shear stress becomes finite at any point and the normal stress possesses only square root singularity at the crack tip. Therefore, the conventional stress intensity factor of the normal stress at the crack tip is used. The numerical illustration of the obtained solution is given.

\section{Introduction}

Interface cracks in many cases are the main reason of composite constructions failure. Therefore, much attention has been paid to the investigation of interface fracture in the framework of the open crack model. Such model is connected with oscillating singularity, which was the most clearly investigated in the paper [1] for a crack between two isotropic materials. The contact zone model, assuming a partial closing of the crack faces and eliminating a physically unreal oscillation, has been suggested in [2] and analytically developed in the papers [3-5].

The problem of an interface crack between two different anisotropic materials is much more complicated and therefore less studied compared to an isotropic case. An analytical analysis of this problem in the framework of an open interface crack model was carried out in [6] and continued in [711]. The phenomenon of oscillation was confirmed in these papers for an anisotropic case. Accounting of the crack faces contact for anisotropic bimaterials has been done in papers $[12,13]$ in an analytically numerical way and in $[14,15]$ analytically.

In many cases the interface is much softer than the adhered matrixes; therefore, thin yield zones develop at the crack tips along the interface. Accounting of such zones was performed in [16] with use of Coulomb law of dry friction and in $[17,18]$ by means of applying Dugdale [19] model for the simulation of the mechanical fields in these zones. However to the author's knowledge a thin yield zone based upon the Treska's-Saint-Venant's theory has been never used for an interface crack in neither orthotropic nor isotropic bimaterial.

In this paper an interface crack with a yield zone based upon maximum shear stress criterion is studied. An exact analytical solution for the associated mathematical model is derived. This solution is free from an oscillation and, therefore, the conventional form of stress fracture criterion can be used. 


\section{Formulation of the Basic Relations}

The constitutive relations of elasticity for a linear anisotropic material in the absence of body forces in a fixed rectangular coordinate system $x_{i}$ can be presented in the form [20]

$$
\begin{aligned}
\sigma_{i j} & =C_{i j k l} u_{k, l}, \\
\sigma_{i j, j} & =0,
\end{aligned}
$$

where $\sigma_{i j}, u_{k}$ are stress and displacements components; $C_{i j k l}$ are the elastic moduli, for which $C_{i j k l}=C_{k l i j}=C_{j i k l}=C_{i j l k}$ hold true and Einstein's summation convention from 1 to 3 for Latin suffixes has been used.

Substituting (1) in (2) one gets the following Lame equations:

$$
C_{i j k l} u_{k, l j}=0
$$

Assuming that all fields are independent of the coordinate $x_{3}$, the solution of (3) according to the method suggested in [21] and developed in [6] can be presented in the form:

$$
u_{k}=a_{k} f\left(x_{1}+p x_{2}\right)
$$

where $f$ is an arbitrary analytic function of the complex variable $z=x_{1}+p x_{2} ; p$ and $a_{1}, a_{2}, a_{3}$ are eigenvalue and eigenvector components of the following system:

$$
\left[\mathbf{Q}+p\left(\mathbf{R}+\mathbf{R}^{T}\right)+p^{2} \mathbf{T}\right] \mathbf{a}=\mathbf{0} .
$$

The elements of the $3 \times 3$ matrices $\mathbf{Q}, \mathbf{R}$ and $\mathbf{T}$ are defined as

$$
\begin{aligned}
& \mathbf{Q}=\left[Q_{i k}\right], \\
& \mathbf{R}=\left[R_{i k}\right], \\
& \mathbf{T}=\left[T_{i k}\right] \\
& \text { with } Q_{i k}=C_{i 1 k 1}, R_{i k}=C_{i 1 k 2}, T_{i k}=C_{i 2 k 2}(i, k=1,2,3) .
\end{aligned}
$$

Here and afterwards the superscript $\mathrm{T}$ stands for the transposed matrix. A nontrivial solution of (5) exists if $p$ is a root of the equation

$$
\operatorname{det}\left[\mathbf{Q}+p\left(\mathbf{R}+\mathbf{R}^{T}\right)+p^{2} \mathbf{T}\right]=0 .
$$

Since (7) has no real roots [9] we denote the roots of this equation with positive imaginary parts as $p_{\alpha}$ and the associated eigenvectors of (5) as $\mathbf{a}_{\alpha}$ (subscript $\alpha$ here and afterwards takes the numerals 1, 2, 3). The most general real solution of (3) can be presented as [9]

$$
\mathbf{U}=\mathbf{A f}(z)+\overline{\mathbf{A f}}(\bar{z}),
$$

where $\mathbf{U}=\left[u_{1}, u_{2}, u_{3}\right]^{T}, \mathbf{A}=\left[\mathbf{a}_{1}, \mathbf{a}_{2}, \mathbf{a}_{3}\right]$ is a matrix composed of eigenvectors, $\mathbf{f}(z)=\left[f_{1}\left(z_{1}\right), f_{2}\left(z_{2}\right), f_{3}\left(z_{3}\right)\right]^{\mathrm{T}}$ is an arbitrary vector function, $z_{\alpha}=x_{1}+p_{\alpha} x_{2}$, and the overbar stands for the complex conjugate. Introducing the vector

$$
\mathbf{t}=\left[\sigma_{12}, \sigma_{22}, \sigma_{32}\right]^{T}
$$

and using (2), this vector can be presented in the form

$$
\mathbf{t}=\mathbf{B} \mathbf{f}^{\prime}(z)+\overline{\mathbf{B f}}^{\prime}(\bar{z})
$$

where the components of $3 \times 3$ matrix $\mathbf{B}$ are defined as

$$
\begin{aligned}
B_{i \alpha}=\left(C_{i 2 k 1}+p_{\alpha} C_{i 2 k 2}\right) & a_{k \alpha} \\
& (\text { not summed over index } \alpha) .
\end{aligned}
$$

Further a bimaterial composed of two different anisotropic semi-infinite spaces $x_{2}>0$ and $x_{2}<0$ with mechanical properties defined by the matrices $C_{i j k l}^{(1)}$ and $C_{i j k l}^{(2)}$, respectively, is considered. We assume that the vector $\mathbf{t}$ is continuous across the whole bimaterial interface and the part $L=\left\{\left(-\infty, c_{1}\right) \cup\left(b_{1}, c_{2}\right) \cup \ldots\left(b_{n}, \infty\right)\right\}$ of the interface $-\infty<x_{1}<\infty, x_{2}=0$ are bounded. That is, the boundary conditions at the interface $x_{2}=0$ are as follows:

$$
\begin{aligned}
& \mathbf{t}^{(1)}\left(x_{1}, 0\right)=\mathbf{t}^{(2)}\left(x_{1}, 0\right) \text { for } x_{1} \in(-\infty, \infty), \\
& \mathbf{U}^{(1)}\left(x_{1}, 0\right)=\mathbf{U}^{(2)}\left(x_{1}, 0\right) \text { for } x_{1} \in L \text {. }
\end{aligned}
$$

Satisfying (12), using for each material the presentations (8), (10), and applying the method written in detail in the paper [14] the following expressions at the interface are obtained:

$$
\begin{aligned}
& \left\langle\mathbf{U}^{\prime}\left(x_{1}\right)\right\rangle=\mathbf{W}^{+}\left(x_{1}\right)-\mathbf{W}^{-}\left(x_{1}\right), \\
& \mathbf{t}^{(1)}\left(x_{1}, 0\right)=\mathbf{G} \mathbf{W}^{+}\left(x_{1}\right)-\overline{\mathbf{G}} \mathbf{W}^{-}\left(x_{1}\right),
\end{aligned}
$$

where $\left\langle\mathbf{U}^{\prime}\left(x_{1}\right)\right\rangle=\mathbf{U}^{\prime(1)}\left(x_{1}, 0\right)-\mathbf{U}^{\prime(2)}\left(x_{1}, 0\right)$ is the jump of the function $\mathbf{U}^{\prime}\left(x_{1}\right)$ across the material interface, $\mathbf{G}=\mathbf{B}^{(1)} \mathbf{D}^{-1}$, $\mathbf{D}=\mathbf{A}^{(1)}-\overline{\mathbf{L}} \mathbf{B}^{(1)}, \mathbf{L}=\mathbf{A}^{(2)}\left(\mathbf{B}^{(2)}\right)^{-1}$.

It should be noted that the vector function $\mathbf{W}(\mathrm{z})=$ $\left[W_{1}(z), W_{2}(z), W_{3}(z)\right]^{T}$ is analytic in the whole plane with a cut along $(-\infty, \infty) \backslash \mathrm{L}$. The relations (14), (15) play an important role for the formulation of various problems of linear relationship for anisotropic bimaterials with cuts at the material interfaces under the action of mechanical loadings.

The main attention in the following will be paid to the consideration of orthotropic materials with the axis of the material symmetry parallel to $x_{3}$ as the most important class of anisotropic materials. In this case the matrix $\mathbf{G}$ has the following structure:

$$
\mathbf{G}=\left[\begin{array}{ccc}
G_{11} & G_{12} & G_{13} \\
G_{21} & G_{22} & G_{23} \\
G_{31} & G_{32} & G_{33}
\end{array}\right]=\left[\begin{array}{ccc}
i g_{11} & g_{12} & 0 \\
g_{21} & i g_{22} & 0 \\
0 & 0 & i g_{33}
\end{array}\right],
$$

where all $g_{i j}$ are real. It is clear that the plane and out of plane problems can be decoupled. Because of the simplicity of the out of plane problem solution our attention will be focused on the plane problem for the displacement components $\left(u_{1}, u_{2}\right)$. In this case similarly to the contracted notations of the anisotropic elasticity [20] the following relations for the elastic coefficients related to the $\left(x_{1}, x_{2}\right)$-plane can be 


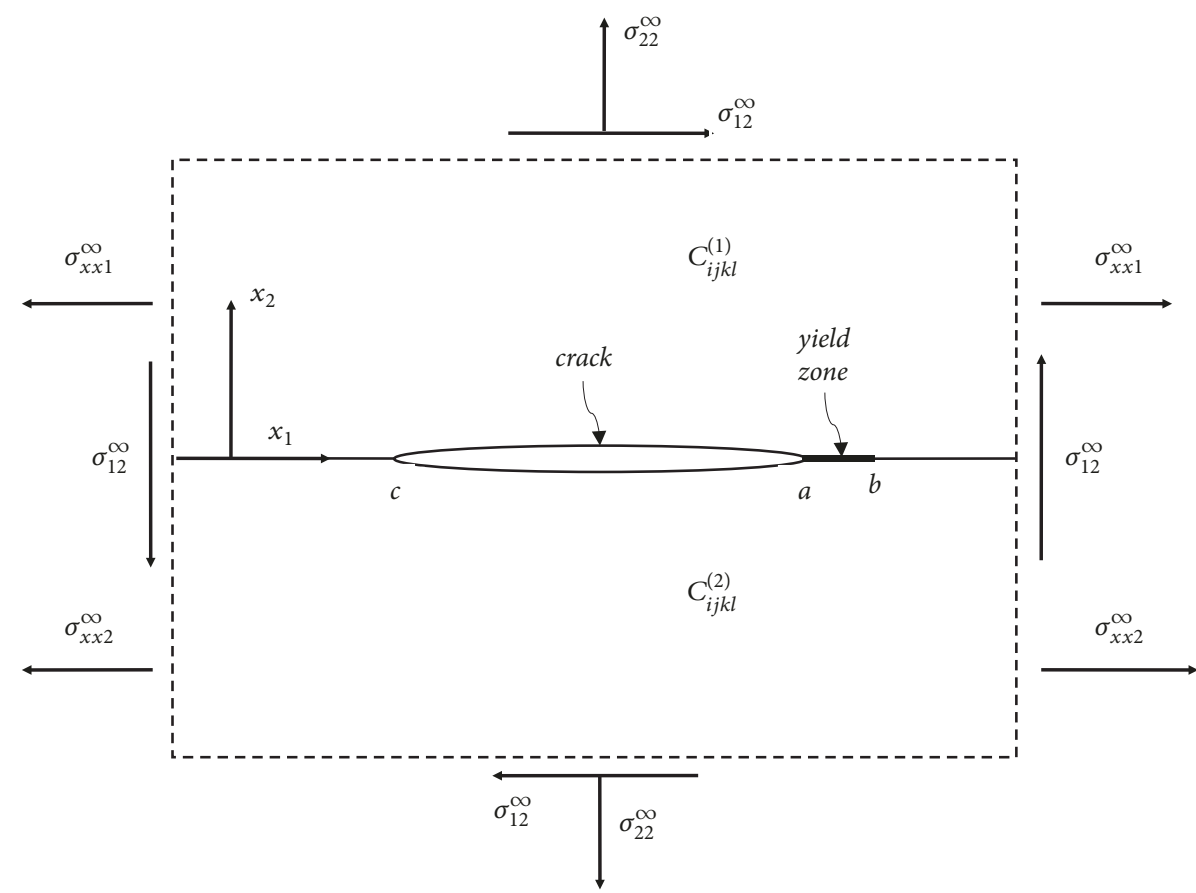

FIGURE 1: A crack between two anisotropic materials with a yield zone.

introduced: $C_{1111}=C_{11}, C_{1122}=C_{12}, C_{2222}=C_{22}, C_{1212}=$ $\mathrm{C}_{44}$ and the combination of each of the first two equations of (14), (15) can be presented in the form (see for details [14])

$$
\begin{aligned}
& \sigma_{22}^{(1)}\left(x_{1}, 0\right)+i m_{1} \sigma_{12}^{(1)}\left(x_{1}, 0\right) \\
& \quad=q_{1}\left[\Omega_{1}^{+}\left(x_{1}\right)+\gamma_{1} \Omega_{1}^{-}\left(x_{1}\right)\right], \quad(\mathrm{j}=1,2), \\
& \left\langle u_{1}^{\prime}\left(x_{1}\right)\right\rangle+i S_{1}\left\langle u_{2}^{\prime}\left(x_{1}\right)\right\rangle=\Omega_{1}^{+}\left(x_{1}\right)-\Omega_{1}^{-}\left(x_{1}\right),
\end{aligned}
$$

where

$$
\begin{aligned}
\Omega_{1}(z) & =W_{1}(z)+i S_{1} W_{2}(z), \\
\gamma_{1} & =-\frac{\left(g_{21}+m_{1} g_{11}\right)}{q_{1}}, \\
S_{1} & =\frac{g_{22}+m_{1} g_{12}}{g_{21}-m_{1} g_{11}}, \\
q_{1} & =g_{21}-m_{1} g_{11}, \\
m_{1} & =-\sqrt{-\frac{g_{21} g_{22}}{g_{11} g_{12}} .}
\end{aligned}
$$

It is worthy to note that the function $\Omega_{1}(z)$ is analytic in the whole plane with a cut along $(-\infty, \infty) \backslash \mathrm{L}$.

\section{A Crack at the Interface of Two Materials}

Let us assume further that a crack takes place at the section $[c$, a] of the material interface. The half-spaces are subjected to uniformly distributed normal stress $\sigma_{22}^{\infty}$ and shear stress $\sigma_{12}^{\infty}$ at infinity, which do not depend on the coordinate $x_{3}$. The crack faces are free of loading. This kind of external fields initiates plane deformation state; therefore, only the crosssection orthogonal to $x_{3}$ (Figure 1) can be considered and the relations (17), (18) with $n=1$ are valid. We do not pay attention for a while to the section $(a, b)$ of the interface, which will need consideration later.

The boundary conditions for the formulated problem are as follows:

$$
\begin{aligned}
\sigma_{21}^{(1)} & =\sigma_{21}^{(2)}=0, \\
\sigma_{22}^{(1)} & =\sigma_{22}^{(2)}=0 \\
\left\langle\sigma_{21}\right\rangle & =0, \\
\left\langle\sigma_{22}\right\rangle & =0, \\
\left\langle u_{1}^{\prime}\right\rangle & =0, \\
\left\langle u_{2}^{\prime}\right\rangle & =0
\end{aligned}
$$

for $x_{1} \notin(c, a)$.

Satisfying conditions (21) and (22) with the use of (17), (18) provides the continuity of the function $\Omega_{1}(z)$ over the segments $x_{1} \notin(c, a)$ of the material interface and also leads to the following equation:

$$
\Omega_{1}^{+}\left(x_{1}\right)+\gamma_{1} \Omega_{1}^{-}\left(x_{1}\right)=0 \text { for } c<x_{1}<a .
$$


The conditions at infinity follow from (17) and can be written in the form

$$
\left.\Omega_{1}(z)\right|_{z \longrightarrow \infty}=\widetilde{\sigma}_{22}+i m_{1} \widetilde{\sigma}_{21}
$$

where $\widetilde{\sigma}_{22}=\sigma_{22}^{\infty} / r_{1}, \widetilde{\sigma}_{21}=\sigma_{21}^{\infty} / r_{1}, r_{1}=\left(1+\gamma_{1}\right) q_{1}$.

The solution of (23) under the condition at infinity (24) has been obtained with use of [22] in the form

$$
\begin{aligned}
& \Omega_{1}(z) \\
& =\left(\widetilde{\sigma}_{22}+i m_{1} \widetilde{\sigma}_{21}\right) \frac{z-(a+c) / 2-i \varepsilon l_{0}}{\sqrt{(z-c)(z-a)}}\left(\frac{z-c}{z-a}\right)^{i \varepsilon}
\end{aligned}
$$

where $\varepsilon=(1 / 2 \pi) \ln \gamma_{1}, . l_{0}=a-c$.

The stresses at the interface are found from (17), (25) as follows:

$$
\begin{array}{r}
\sigma_{22}^{(1)}\left(x_{1}, 0\right)+i m_{1} \sigma_{21}^{(1)}\left(x_{1}, 0\right) \\
=\left(\sigma_{22}^{\infty}+i m_{1} \sigma_{21}^{\infty}\right) \frac{x_{1}-(a+c) / 2-i \varepsilon l_{0}}{\sqrt{\left(x_{1}-c\right)\left(x_{1}-a\right)}}\left(\frac{x_{1}-c}{x_{1}-a}\right)^{i \varepsilon} \\
\text { for } x_{1}>a .
\end{array}
$$

The derivative of the displacement jumps is obtained by use of the formula (18) in the form

$$
\begin{gathered}
\left\langle u_{1}{ }^{\prime}\left(x_{1}, 0\right)\right\rangle+i S_{1}\left\langle u_{2}{ }^{\prime}\left(x_{1}, 0\right)\right\rangle=-\frac{\left(\sigma_{22}^{\infty} i-m_{1} \sigma_{21}^{\infty}\right)}{q_{1} \sqrt{\gamma_{1}}} \\
\cdot \frac{\left(x_{1}-(a+c) / 2-i \varepsilon l_{0}\right)}{\sqrt{\left(x_{1}-c\right)\left(a-x_{1}\right)}}\left(\frac{x_{1}-c}{a-x_{1}}\right)^{i \varepsilon}
\end{gathered}
$$

$$
\text { for } c<x_{1}<a \text {. }
$$

After integrating the last relation, we obtain

$$
\begin{aligned}
& \left\langle u_{1}\left(x_{1}, 0\right)\right\rangle+i S_{1}\left\langle u_{2}\left(x_{1}, 0\right)\right\rangle \\
& =\sqrt{\left(x_{1}-c\right)\left(a-x_{1}\right)}\left\{\frac{\left(\sigma_{22}^{\infty} i-m_{1} \sigma_{21}^{\infty}\right)}{q_{1} \sqrt{\gamma_{1}}}\left(\frac{x_{1}-c}{a-x_{1}}\right)^{i \varepsilon}\right\} \\
& \text { for } c<x_{1}<a .
\end{aligned}
$$

\section{Formulation of the Problem and Development of the Interface Crack Model Free from Oscillation}

It is clearly seen from (26), (27) that the mechanical stresses and the derivative of displacement jumps are singular at the crack tips. Moreover this singularity is oscillating. To remove this oscillation different models were suggested, for example, $[2,17,18]$. In the present paper the model based upon the introduction of the shear yield zones is suggested. As it will be shown later, either both of these zones are very short or one zone is substantially shorter than the other one; therefore their mutual influence can be neglected. Taking into account this circumstance we will consider only the longer zone ( $a$, b) for simplicity, assuming that it occurs at the right crack tip (Figure 1). If the longer zone occurs at another crack tip then this zone can be considered by simple transformation of halfspaces.

Thus the boundary conditions for the considered model can be written as follows:

$$
\begin{aligned}
\sigma_{21}^{(1)} & =\sigma_{21}^{(2)}=0, \\
\sigma_{22}^{(1)} & =\sigma_{22}^{(2)}=0 \\
\quad \text { for } c<x_{1}<a, & \\
\sigma_{21}^{(1)} & =\sigma_{21}^{(2)}=\tau_{S}, \\
\left\langle\sigma_{22}\right\rangle & =0, \\
\left\langle u_{2}^{\prime}\right\rangle & =0
\end{aligned}
$$

for $a<x_{1}<b$,

$$
\begin{aligned}
& \left\langle\sigma_{21}\right\rangle=0, \\
& \left\langle\sigma_{22}\right\rangle=0, \\
& \left\langle u_{1}^{\prime}\right\rangle=0, \\
& \left\langle u_{2}^{\prime}\right\rangle=0
\end{aligned}
$$

for $x_{1} \notin(c, b)$.

where $\tau_{S}$ is the shear yield limit of the adhesive layer.

Satisfying the interface conditions (29) and using (17) one gets (23). Additionally the first and third conditions (30) lead to the equations

$$
\begin{aligned}
\operatorname{Im}\left[\Omega_{1}^{+}\left(x_{1}\right)+\gamma_{1} \Omega_{1}^{-}\left(x_{1}\right)\right] & =-\frac{m_{1} \tau_{s}}{q_{1}}, \\
\operatorname{Im}\left[\Omega_{1}^{+}\left(x_{1}\right)-\Omega_{1}^{-}\left(x_{1}\right)\right] & =0
\end{aligned}
$$

$$
\text { for } a<x_{1}<b \text {. }
$$

Satisfaction of the boundary conditions (31) provides the analyticity of the function $\Omega_{1}(z)$ outside of the interval $(c, b)$ and the last relations lead to the equation

$$
\operatorname{Im} \Omega_{1}^{ \pm}\left(x_{1}\right)=\tau \text { for } a<x_{1}<b,
$$

where $\tau=-m_{1} \tau_{S} / r_{1}$.

Equations (23) and (33) present the nonhomogeneous combined Dirichlet-Riemann boundary value problem. The conditions at infinity (24) are valid for this problem also. By using the results of the paper by Nahmein and Nuller [23] the general solution of the homogeneous problem corresponding to (23), (33) can be presented in the form

$$
\Omega_{1 h}(z)=P(z) E_{1}(z)+Q(z) E_{2}(z),
$$

where

$$
\begin{aligned}
& P(z)=C_{1} z+C_{2}, \\
& Q(z)=D_{1} z+D_{2} .
\end{aligned}
$$


The functions

$$
\begin{aligned}
& E_{1}(z)=\frac{i e^{i \varphi(z)}}{\sqrt{(z-c)(z-b)}} \\
& E_{2}(z)=\frac{e^{i \varphi(z)}}{\sqrt{(z-c)(z-a)}}
\end{aligned}
$$

present the canonical solutions of the homogeneous problem (23), (33), where $\varphi(z)=2 \varepsilon \ln (\sqrt{(b-a)(z-c)} /(\sqrt{l(z-a)}+$ $\sqrt{(a-c)(z-b)})), l=b-c$, and $C_{1}, C_{2}, D_{1}, D_{2}$ are arbitrary real coefficients.

A particular solution of the nonhomogeneous combined Dirichlet-Riemann boundary value problem for certain right sides of (23) and (33) was analyzed in [24]. In the present case of the problem (23), (33) its particular solution can be found in the form

$$
\Omega_{1 p}(z)=\Phi(z) E_{1}(z)
$$

where $\Phi(z)$ is assumed to be analytic in the whole complex plane with a cut $[a, b]$ along the $x_{1}$-axis. It should be mentioned that $\Omega_{1 p}(z)$ satisfies (33). Substituting (37) into (33) and taking into account that $\operatorname{Im} E_{1}^{ \pm}\left(x_{1}\right)=0$ on $(a, b)$ one has the following equation:

$$
\operatorname{Im} \Phi^{ \pm}\left(x_{1}\right)=\psi^{ \pm}\left(x_{1}\right) \text { for } a<x_{1}<b,
$$

where $\psi\left(x_{1}\right)=\tau / E_{1}\left(x_{1}\right)$.

A solution of the Dirichlet problem (38) has the following form [25, formula (46.25)]:

$$
\begin{aligned}
\Phi(z)= & \frac{Y(z)}{2 \pi} \int_{a}^{b} \frac{\psi^{+}(t)+\psi^{-}(t)}{Y^{+}(t)(t-z)} d t \\
& +\frac{1}{2 \pi} \int_{a}^{b} \frac{\psi^{+}(t)-\psi^{-}(t)}{t-z} d t,
\end{aligned}
$$

where $Y(z)=\sqrt{(z-a)(z-b)}$ and $0 \leq \arg (z-a) \leq 2 \pi, 0 \leq$ $\arg (z-b) \leq 2 \pi$.

Using

$$
\begin{aligned}
\psi^{+}(t)+\psi^{-}(t) & =-2 \tau \sqrt{(t-c)(b-t)} \sinh \varphi_{0}(t), \\
\psi^{+}(t)-\psi^{-}(t) & =2 \tau \sqrt{(t-c)(b-t)} \cosh \varphi_{0}(t) \\
\varphi_{0}\left(x_{1}\right) & =2 \varepsilon \tan ^{-1} \sqrt{\frac{(a-c)\left(b-x_{1}\right)}{(b-c)\left(x_{1}-a\right)}} \\
Y^{+}(\mathrm{t}) & =-i \sqrt{(t-a)(b-t)}
\end{aligned}
$$

$$
\text { at }(a, b) \text {, }
$$

the formula (39) takes the form

$$
\Phi(z)=\frac{\tau}{\pi}\left[-i Y(z) L_{1}(z)+L_{2}(z)\right],
$$

where $L_{1}(z)=\int_{a}^{b} \sqrt{(t-c) /(t-a)}\left(\sinh \varphi_{0}(t) /(t-z)\right) d t$, $L_{2}(z)=\int_{a}^{b} \sqrt{(t-c)(b-t)}\left(\cosh \varphi_{0}(t) /(t-z)\right) d t$.
The general solution of the problem (23), (33) can be found by summing the solutions (34) and (37). Arbitrary constants $C_{1}, C_{2}, D_{1}, D_{2}$ can be found from the condition at infinity (24) together with condition of the displacement uniqueness which due to (18) can be written in the form

$$
\int_{c}^{b}\left(\Omega_{1}^{+}\left(x_{1}\right)-\Omega_{1}^{-}\left(x_{1}\right)\right) d x_{1}=0 .
$$

Considering that for the validity of last equation the coefficient before $z^{-1}$ in the expansion of $\Omega_{1}(z)$ at infinity should be equal to zero [24] and also

$$
\begin{aligned}
\left.E_{1}(z)\right|_{z \rightarrow \infty} & =i z^{-2} e^{i \beta}\left(z+i \beta_{1}+\frac{c+b}{2}\right)+o\left(z^{-3}\right), \\
\left.E_{2}(z)\right|_{z \rightarrow \infty} & =z^{-2} e^{i \beta}\left(z+i \beta_{1}+\frac{c+a}{2}\right)+o\left(z^{-3}\right), \\
\left.\Phi(z)\right|_{z \rightarrow \infty} & =-i R+o\left(z^{-1}\right), \\
R & =\frac{\tau}{\pi} \int_{a}^{b} \sqrt{\frac{t-c}{t-a}} \sinh \varphi_{0}(t) d t
\end{aligned}
$$

one gets the following expressions for the unknown coefficients

$$
\begin{aligned}
& C_{1}=-\widetilde{\sigma}_{23} \sin \beta-\widetilde{E}_{1} \cos \beta, \\
& D_{1}=\widetilde{\sigma}_{23} \cos \beta-\widetilde{E}_{1} \sin \beta, \\
& C_{2}=-\frac{c+b}{2} C_{1}-\beta_{1} D_{1}, \\
& D_{2}=\beta_{1} C_{1}-\frac{c+a}{2} D_{1}-R,
\end{aligned}
$$

where $\beta=\varepsilon \ln ((1-\sqrt{1-\lambda}) /(1+\sqrt{1-\lambda})), \quad \beta_{1}=$ $\varepsilon \sqrt{(a-c)(b-c)}$,

$$
\lambda=\frac{b-a}{b-c} .
$$

Using the obtained solutions the general solution of the nonhomogeneous combined Dirichlet-Riemann boundary value problem (23), (33) can be presented in the form

$$
\Omega_{1}(z)=(P(z)+\Phi(z)) E_{1}(z)+Q(z) E_{2}(z) .
$$

From this solution all required quantities at the material interface can be found.

\section{Stress Intensity Factor and Yield Zone Length}

According to (17) the stress field on the right side from the yield zone can be presented in the form:

$$
\begin{aligned}
& \sigma_{22}^{(1)}\left(x_{1}, 0\right)+i m_{1} \sigma_{21}^{(1)}\left(x_{1}, 0\right) \\
& \quad=r_{1}\left\{\left(P\left(x_{1}\right)+\Phi\left(x_{1}\right)\right) E_{1}\left(x_{1}\right)+Q\left(x_{1}\right) E_{2}\left(x_{1}\right)\right\} .
\end{aligned}
$$


For an arbitrary position of the point $b$, which defines the yield zone length, the right hand side of (47) is singular for $x_{1} \longrightarrow b+0$ and besides $P(b)+\Phi(b)$ is real and $E_{1}\left(x_{1}\right)=$ $i / \sqrt{(b-c)\left(x_{1}-b\right)}$ is pure imaginary. Therefore, for any $b$ the normal stress $\sigma_{22}^{(1)}\left(x_{1}, 0\right)$ is finite for $x_{1} \longrightarrow b+0$ whilst $\sigma_{12}^{(1)}\left(x_{1}, 0\right)$ is singular. For removing of this singularity the equation

$$
P(b)+\Phi(b)=0
$$

should be valid. After some transformation this equation can be written as follows:

$$
\begin{array}{r}
m_{1} \delta \cos \beta-\sin \beta-2 \varepsilon \sqrt{1-\lambda}\left(\cos \beta+m_{1} \delta \sin \beta\right) \\
-\frac{2 m_{1}}{\pi(b-c)} \frac{\tau_{S}}{\sigma_{22}^{\infty}} \int_{a}^{b} \sqrt{\frac{t-c}{b-t}} \cosh \varphi_{0}(t) d t=0 .
\end{array}
$$

where $\delta=\sigma_{21}^{\infty} / \sigma_{22}^{\infty}$.

This equation should be solved with respect to $\lambda$ and after that the position of the point $b$ can be found from (45). Usually (49) can be solved numerically and that allows finding the largest root of this equation from the interval $(0,1)$ which we denote $\lambda_{0}$.

The normal stress at the interval $(a, b)$ according to (17), (46) can be found in the form

$$
\sigma_{22}^{(1)}\left(x_{1}, 0\right)=q_{1}\left[\Omega_{1}^{+}\left(x_{1}\right)+\gamma_{1} \Omega_{1}^{-}\left(x_{1}\right)\right]-i m_{1} \tau_{S} .
$$
[24]

Substituting the formula (46), taking into account that

$$
\begin{aligned}
& E_{1}^{ \pm}\left(x_{1}\right)=\frac{ \pm e^{ \pm \phi_{0}\left(x_{1}\right)}}{\sqrt{\left(x_{1}-c\right)\left(b-x_{1}\right)}}, \\
& E_{2}^{ \pm}\left(x_{1}\right)=\frac{e^{ \pm \phi_{0}\left(x_{1}\right)}}{\sqrt{\left(x_{1}-c\right)\left(x_{1}-a\right)}}
\end{aligned}
$$

$$
\text { for } x_{1} \in(a, b)
$$

and using Plemeli formulas [22] one gets the following expression:

$$
\begin{aligned}
& q_{1}^{-1} \sigma_{22}^{(1)}\left(x_{1}, 0\right)=P\left(x_{1}\right) \frac{e^{\varphi_{0}\left(x_{1}\right)}-\gamma_{1} e^{-\varphi_{0}\left(x_{1}\right)}}{\sqrt{\left(x_{1}-c\right)\left(b-x_{1}\right)}}+Q\left(x_{1}\right) \\
& \cdot \frac{e^{\varphi_{0}\left(x_{1}\right)}+\gamma_{1} e^{-\varphi_{0}\left(x_{1}\right)}}{\sqrt{\left(x_{1}-c\right)\left(x_{1}-\mathrm{a}\right)}} \\
& +\frac{\tau}{\pi}\left\{-\sqrt{\frac{x_{1}-a}{x_{1}-c}\left[e^{\varphi_{0}\left(x_{1}\right)}+\gamma_{1} e^{-\varphi_{0}\left(x_{1}\right)}\right] L_{1}\left(x_{1}\right)}\right. \\
& \left.+\frac{e^{\varphi_{0}\left(x_{1}\right)}-\gamma_{1} e^{-\varphi_{0}\left(x_{1}\right)}}{\sqrt{\left(x_{1}-c\right)\left(b-x_{1}\right)}} L_{2}\left(x_{1}\right)\right\},
\end{aligned}
$$

where the integrals $L_{1}\left(x_{1}\right)$ and $L_{2}\left(x_{1}\right)$ should be considered here in sense of principal value on Cauchy [25].

Consider further the stress intensity factor (SIF) of the normal stress at the point $a$

$$
K_{1}=\lim _{x_{1} \longrightarrow a+0} \sqrt{2 \pi\left(x_{1}-a\right)} \sigma_{22}^{(1)}\left(x_{1}, 0\right) .
$$

Considering that $L_{1}\left(x_{1}\right)$ has a square root singularity for $x_{1} \longrightarrow a+0$ and $L_{2}\left(x_{1}\right)$ has the logarithmic singularity at this point we get the following formula:

$$
K_{1}=2 q_{1} \sqrt{2 \pi \gamma_{1}} \frac{Q(a)}{\sqrt{a-c}} .
$$

This formula after some transformations takes the form

$$
\begin{aligned}
& K_{1} \\
& \quad=\frac{2 q_{1} \sqrt{2 \pi \gamma_{1}}}{\sqrt{a-c}}\left[\frac{l}{2} \sqrt{1-\lambda}\left(2 \varepsilon C_{1}+\sqrt{1-\lambda} D_{1}\right)-R\right] .
\end{aligned}
$$

The derivative of the crack faces displacement jump at the interval $(c, a)$ (crack opening) can be found due to (18) in the form

$$
S_{1}\left\langle u_{2}^{\prime}\left(x_{1}, 0\right)\right\rangle=\operatorname{Im}\left[\Omega_{1}^{+}\left(x_{1}\right)-\Omega_{1}^{-}\left(x_{1}\right)\right] .
$$

Substituting the expression (46) one gets

$$
\begin{aligned}
& \left\langle u_{2}^{\prime}\left(x_{1}, 0\right)\right\rangle \\
& =\frac{\gamma_{1}+1}{S_{1} \sqrt{\gamma_{1}}}\left[\frac{P\left(x_{1}\right)+\Phi\left(x_{1}\right)}{\sqrt{b-x_{1}}}-i \frac{Q\left(x_{1}\right)}{\sqrt{a-x_{1}}}\right] \\
& . \frac{\exp \left[i \varphi^{*}\left(x_{1}\right)\right]}{\sqrt{x_{1}-c}} \text { for } c<x_{1}<a,
\end{aligned}
$$

where $\varphi^{*}\left(x_{1}\right)=2 \varepsilon \ln \left(\sqrt{(b-a)\left(x_{1}-c\right)} /\left(\sqrt{l\left(a-x_{1}\right)}+\right.\right.$ $\left.\left.\sqrt{(a-c)\left(b-x_{1}\right)}\right)\right)$.

The crack opening can be found by using the following formula:

$$
\left\langle u_{2}\left(x_{1}, 0\right)\right\rangle=\int_{c}^{x_{1}}\left\langle u_{2}^{\prime}(t, 0)\right\rangle d t
$$

\section{Numerical Results and Discussion}

The calculations were performed for a bimaterial composed of boron-epoxy orthotropic material (upper one) with $C_{11}^{(1)}=$ $26.9 C_{66}^{(1)}, C_{22}^{(1)}=3.6 C_{66}^{(1)}, C_{12}^{(1)}=3.15 C_{66}^{(1)}, C_{66}^{(1)}=4.78 \times 10^{10} \mathrm{~Pa}$ and isotropic material (lower one) having the following characteristics: $\mu^{(2)}=0.478 \times 10^{10} \mathrm{~Pa}, \nu^{(2)}=0.345 . \tau_{S}=$ $10^{7} \mathrm{~Pa}, \mathrm{c}=-10 \mathrm{~mm}, b=10 \mathrm{~mm}$ were chosen and different values of the external mechanical loadings were considered.

In Table 1 the yield zone lengths and the SIF $K_{1}$ are presented for $\sigma_{12}^{\infty}=0$ and different values of $\sigma_{22}^{\infty}$. It can be seen from these results that growing of $\sigma_{22}^{\infty}$ leads to increasing of $\lambda_{0}$ and $K_{1}$. Such conclusion completely agrees with the finding which follows from simple physical inferences. 
TABLE 1: Yield zone length and SIF $K_{1}$ for $\sigma_{12}^{\infty}=0$ and different values of $\sigma_{22}^{\infty}$.

\begin{tabular}{lcccrr}
\hline $10^{-6} \sigma_{22}^{\infty}[\mathrm{Pa}]$ & 1 & 2 & 3 & 5 & 5 \\
\hline $100 \lambda_{0}$ & 0.1318 & 0.3700 & 0.6581 & 0.9750 & \\
\hline $10^{-5} \mathrm{~K}_{1}\left[\mathrm{~Pa} / \mathrm{m}^{3 / 2}\right]$ & 1.644 & 3.396 & 5.161 & 6.931 & 8.704 \\
\hline
\end{tabular}

TABLE 2: Yield zone length and SIF $K_{1}$ for $\sigma_{22}^{\infty}=3 \times 10^{6} \mathrm{~Pa}$ and different values of $\sigma_{12}^{\infty}$.

\begin{tabular}{lccccccc}
\hline $10^{-6} \sigma_{12}^{\infty}[\mathrm{Pa}]$ & 0 & 1 & 2 & 3 & 4 & 5 & 6 \\
\hline $100 \lambda_{0}$ & 0.6581 & 1.902 & 4.039 & 7.100 & 11.04 & 15.77 \\
\hline $10^{-5} \mathrm{~K}_{1}\left[\mathrm{~Pa} / \mathrm{m}^{3 / 2}\right]$ & 5.161 & 5.049 & 5.107 & 5.290 & 5.576 & 5.954 \\
\hline
\end{tabular}

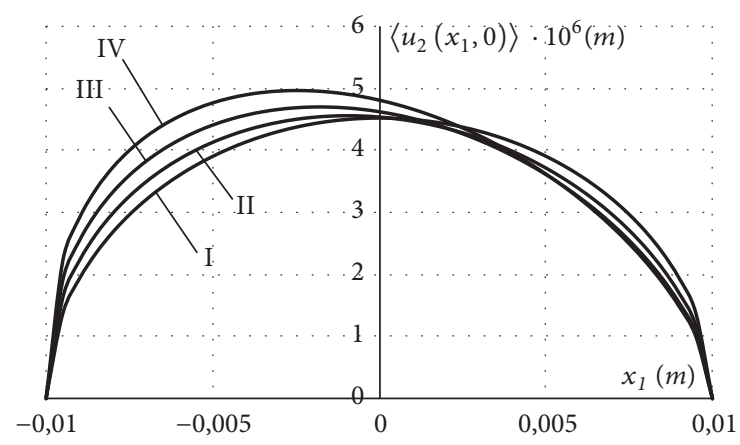

FIGURE 2: Crack opening $10^{6}\left\langle u_{2}\left(x_{1}, 0\right)\right\rangle m$ for $\sigma_{22}^{\infty}=3 \times 10^{6} \mathrm{~Pa}$ and different values of $\sigma_{12}^{\infty}$.

The dependence of the same variables as in Table 1 with respect to the intensity of the shear stress at infinity is given in Table 2. It can be seen that the yield zone length essentially grows with growing of the mentioned stress; however the SIF remain slightly dependent on this parameter. It is also explainable that the applied shear stress does not greatly influence the SIF of the normal stress.

The crack opening $\left\langle u_{2}\left(x_{1}, 0\right)\right\rangle$ for $c=-10 \mathrm{~mm}, b=$ $10 \mathrm{~mm}, \sigma_{22}^{\infty}=3 \times 10^{6} \mathrm{~Pa}$ is presented in Figure 2 for different values of $\sigma_{12}^{\infty}$. Lines I, II, III, and IV correspond to $\sigma_{12}^{\infty}=0,2 \times$ $10^{6} \mathrm{~Pa}, 4 \times 10^{6} \mathrm{~Pa}$ and $6 \times 10^{6} \mathrm{~Pa}$, respectively. It follows from these results that the crack opening is almost symmetrical for $\sigma_{12}^{\infty}=0$ because the yield zone length is extremely small in this case and the problem is almost symmetrical. However, increasing of $\sigma_{12}^{\infty}$ leads to some contortion of the curve and its deviation from the symmetrical state.

The variation of the shear stress $\sigma_{21}^{(1)}\left(x_{1}, 0\right)$ in the right hand side of the yield area for $\sigma_{22}^{\infty}=3 \times 10^{6} \mathrm{~Pa}$, the same crack geometry, mechanical loading and $\sigma_{12}^{\infty}$ equals to 0 (line I), $2 \times 10^{6} \mathrm{~Pa}$ (II), $4 \times 10^{6} \mathrm{~Pa}$ (III) and $6 \times 10^{6} \mathrm{~Pa}$ (IV) is presented in Figure 3. The values of $b$ for the lines I, II, III, and IV are found with use of Table 1 and are equal to $10.13 \mathrm{~mm}$, $10.84 \mathrm{~mm}, 12.48 \mathrm{~mm}$, and $15.38 \mathrm{~mm}$, respectively. As it can be seen the considered yield model eliminated the singularities of the shear stress and the oscillation of the normal stress at the right crack tip. Thereby such approach allows getting shear stress field at the point $b$ free from singularities and transforming the oscillating singularity of the normal stress into conventional square root singularity at the point $a$. The

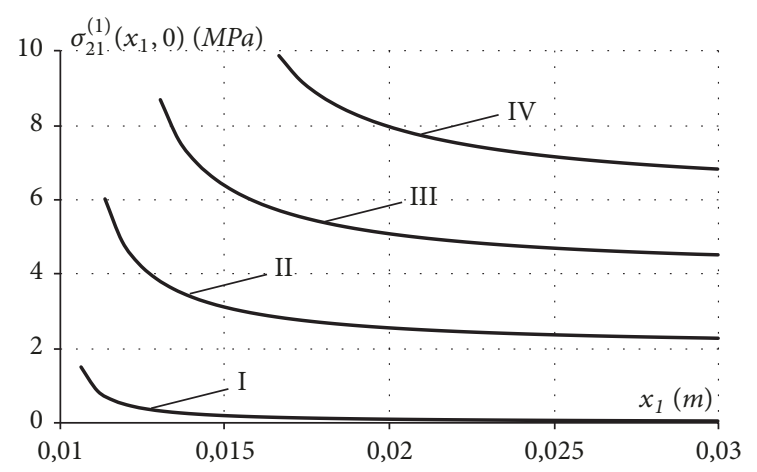

FIgURE 3: Variation of the shear stress $\sigma_{21}^{(1)}\left(x_{1}, 0\right) M P a$ in the right hand side of the yield area for $\sigma_{22}^{\infty}=3 \times 10^{6} \mathrm{~Pa}$ and different values of $\sigma_{12}^{\infty}$.

last circumstance gives the possibility to introduce the SIF of the normal stress in a commonly used form.

\section{Conclusion}

An interface crack in a bimaterial anisotropic space under tensile-shear loading at infinity is considered. Plane strain conditions are assumed and the crack faces are free of stresses. Due to complex potentials approach an exact analytical solution (25) of the formulated problem is given. The stresses and the derivatives of the displacement jumps obtained from this solution have the oscillating square root singularities at the crack tips. The new model based upon the introduction of the shear yield zones at the crack tips is suggested for removing of these singularities. This model is used under the assumption that the interface adhesive layer is softer than the surrounding matrixes. The problem is reduced to the nonhomogeneous combined Dirichlet-Riemann boundary value problem (23), (33) with the conditions at infinity (24). An exact analytical solution of this problem is presented for the case of a single yield zone. The assumption concerning consideration of a single yield zone is approved by the fact that another zone is extremely short and does not influence the longer zone. The length of yield zone is found from the finiteness of the shear stress at its end. This gives the simple transcendental equation (49) for the determination of the yield zone length. 
As a result of the performed modeling the shear stress becomes restricted in the whole region and the normal stress has only square root singularity at the crack tip. It means that the stress intensity factor of the normal stress can be introduced and calculated in the conventional way. The dependencies of the mentioned stress intensity factor, yield zone length, crack opening, and the shear stress on the applied loading are illustrated in the tables and graphical forms. It is particularly shown that the stress intensity factor of the normal stress essentially depends on the normal applied stress and moderately on the external shear stress. Finally, it is worthy to mention that the results of the paper are obtained in a simple analytical form which is convenient for the engineering applications.

\section{Data Availability}

The data obtained in the published article can be used for future investigation on the fracture mechanics of composite materials related to an interface crack in anisotropic and isotropic materials. The data is available upon request.

\section{Conflicts of Interest}

The authors declare that there are no conflicts of interest regarding the publication of this paper.

\section{References}

[1] J. R. Rice, "Elastic fracture mechanics concepts for interfacial cracks," Journal of Applied Mechanics, vol. 55, no. 1, pp. 98-103, 1988.

[2] M. Comninou, "The interface crack," Journal of Applied Mechanics, vol. 44, no. 4, pp. 631-636, 1977.

[3] C. Atkinson, "The interface crack with a contact zone (an analytical treatment)," International Journal of Fracture, vol. 18, no. 3, pp. 161-177, 1982.

[4] I. V. Simonov, "An interface crack in an inhomogeneous stress field," International Journal of Fracture, vol. 46, no. 3, pp. 223235, 1990.

[5] J. Dundurs and A. K. Gautesen, "An opportunistic analysis of the interface crack," International Journal of Fracture, vol. 36, no. 2, pp. 151-159, 1988.

[6] D. L. Clements, "A crack between dissimilar anisotropic media," International Journal of Engineering Science, vol. 9, no. 2, pp. 257-265, 1971.

[7] T. C. Ting, "Explicit solution and invariance of the singularities at an interface crack in anisotropic composites," International Journal of Solids and Structures, vol. 22, no. 9, pp. 965-983, 1986.

[8] K. Wu, "Stress Intensity Factor and Energy Release Rate for Interfacial Cracks Between Dissimilar Anisotropic Materials," Journal of Applied Mechanics, vol. 57, no. 4, p. 882, 1990.

[9] C. Hwu, "Explicit solutions for collinear interface crack problems," International Journal of Solids and Structures, vol. 30, no. 3, pp. 301-312, 1993.

[10] C. Hwu, "Fracture parameters for the orthotropic bimaterial interface cracks," Engineering Fracture Mechanics, vol. 45, no. 1, pp. 89-97, 1993.

[11] W. Qian and C. T. Sun, "Methods for calculating stress intensity factors for interfacial cracks between two orthotropic solids,"
International Journal of Solids and Structures, vol. 35, no. 25, pp. 3317-3330, 1998.

[12] S. S. Wang and I. Choi, "The interface crack between dissimilar anisotropic composite materials," Journal of Applied Mechanics, vol. 50, no. 1, pp. 169-178, 1983.

[13] S. S. Wang and I. Choi, "The crack behavior in dissimilar anisotropic composites under mixed-mode loading," Journal of Applied Mechanics, vol. 50, no. 1, pp. 169-178, 1983.

[14] K. P. Herrmann and V. V. Loboda, "On interface crack models with contact zones situated in an anisotropic bimaterial," Archive of Applied Mechanics, vol. 69, no. 5, pp. 317-335, 1999.

[15] K. P. Herrmann and V. V. Loboda, "Contact zone models for an interface crack in a thermomechanically loaded anisotropic bimaterial," Journal of Thermal Stresses, vol. 24, no. 5, pp. 479506, 2001.

[16] A. A. Kaminskii, L. A. Kipnis, and V. A. Kolmakova, "Slip lines at the end of a cut at the interface of different media," International Applied Mechanics, vol. 31, no. 6, pp. 491-495, 1995.

[17] A. A. Kaminskii, L. A. Kipnis, and V. A. Kolmakova, "On the Dugdaill model for a crack at the interface of different media," International Applied Mechanics, vol. 35, no. 1, pp. 58-63, 1999.

[18] V. V. Loboda and A. E. Sheveleva, "Determining prefracture zones at a crack tip between two elastic orthotropic bodies," International Applied Mechanics, vol. 39, no. 5, pp. 566-572, 2003.

[19] D. S. Dugdale, "Yielding of steel sheets containing slits," Journal of the Mechanics and Physics of Solids, vol. 8, no. 2, pp. 100-104, 1960.

[20] I. S. Sokolnikoff, Mathematical Theory of Elasticity, McGrawHill, New York, NY, USA, 1956.

[21] J. D. Eshelby, W. T. Read, and W. Shockley, "Anisotropic elasticity with applications to dislocation theory," Acta Metallurgica et Materialia, vol. 1, no. 3, pp. 251-259, 1953.

[22] N. I. Muskhelishvili, Some Basic Problems of the Mathematical Theory of Elasticity, Noordhoff International Publishing, Leyden, IL, USA, 1977.

[23] E. L. Nakhmein and B. M. Nuller, "Contact between an elastic half-plane and a partly separated stamp," Journal of Applied Mathematics and Mechanics, vol. 50, no. 4, pp. 507-515, 1986.

[24] K. P. Herrmann and V. V. Loboda, "Fracture mechanical assessment of interface cracks with contact zones in piezoelectric bimaterials under thermoelectromechanical loadings I. Electrically permeable interface cracks," International Journal of Solids and Structures, vol. 40, no. 16, pp. 4191-4217, 2003.

[25] F. D. Gakhov, Boundary Value Problems, Pergamon Press, Oxford, UK, 1966. 


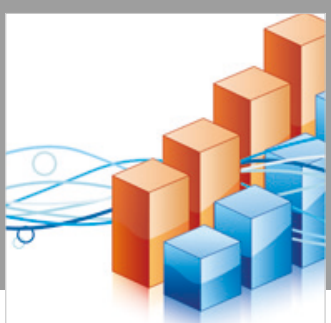

Advances in

Operations Research

\section{-n-m}
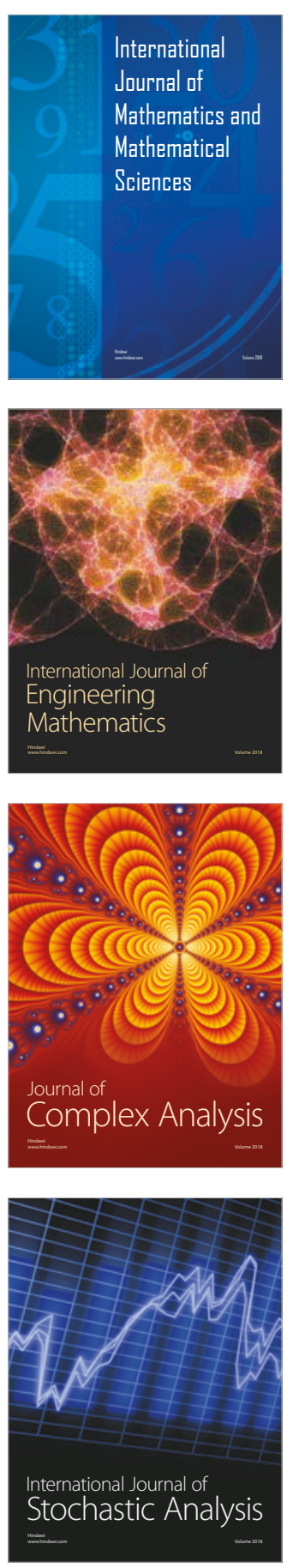
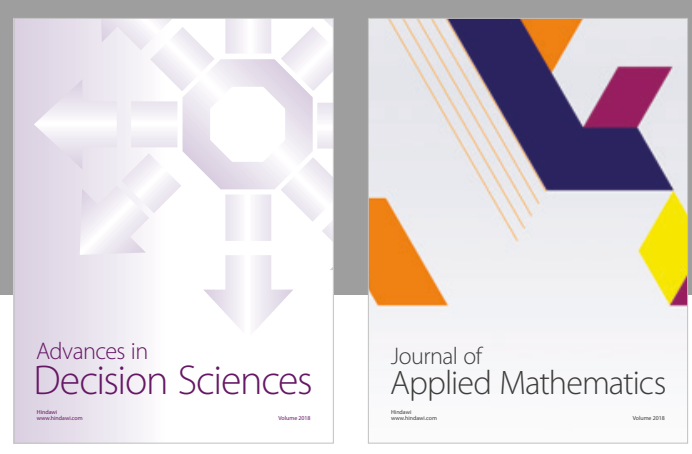

Journal of

Applied Mathematics
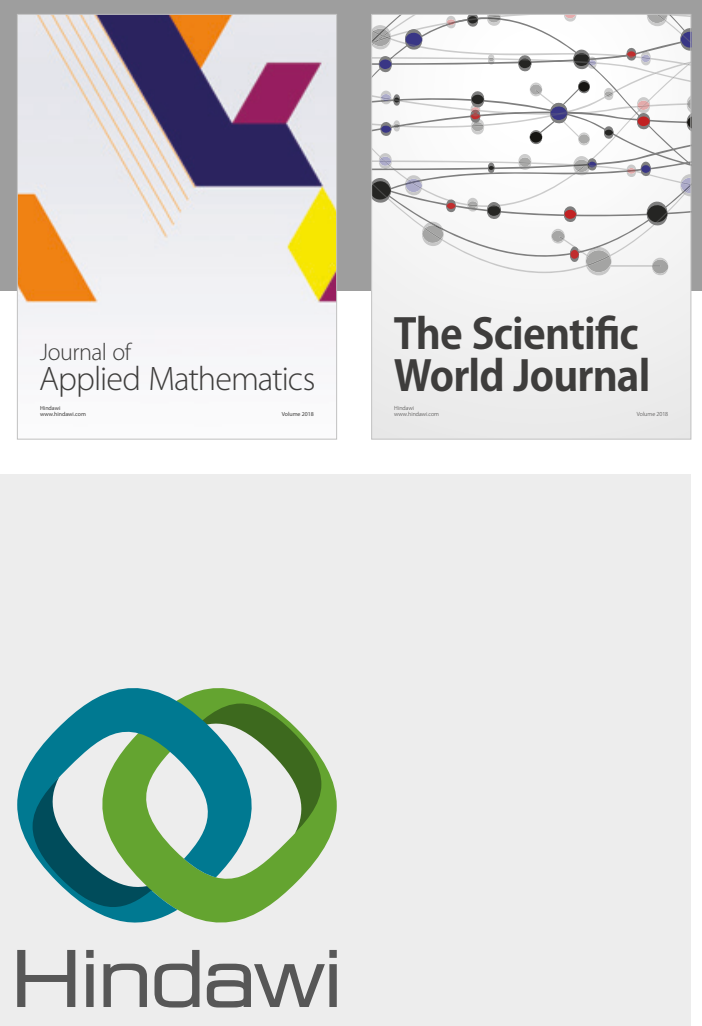

Submit your manuscripts at

www.hindawi.com

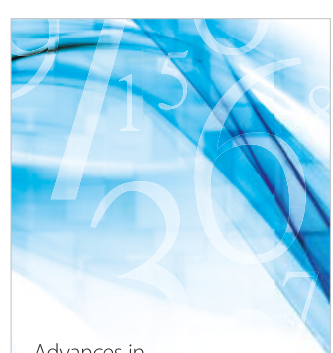

Advances in
Numerical Analysis
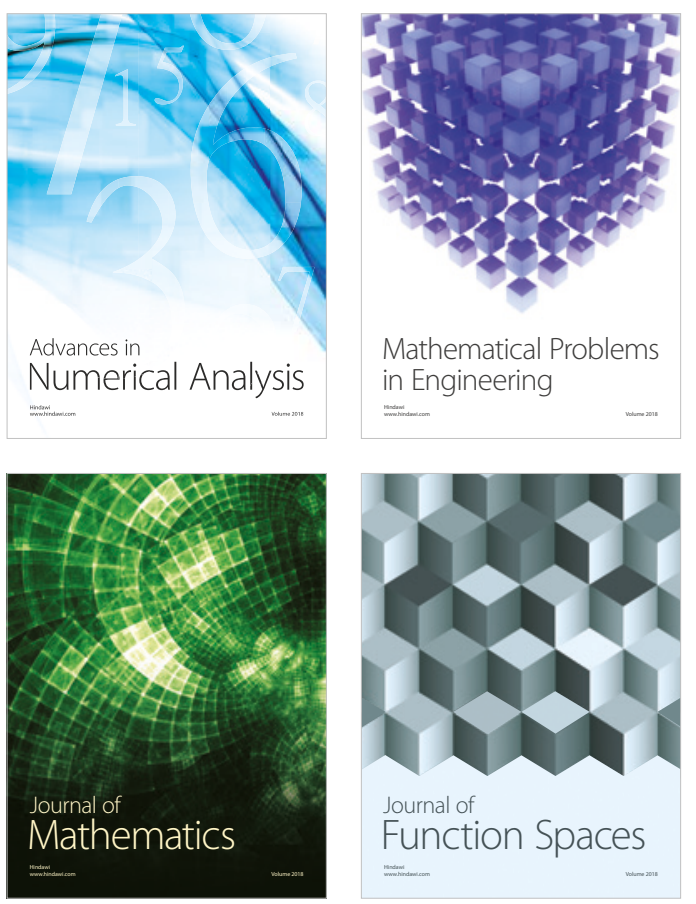

Mathematical Problems in Engineering

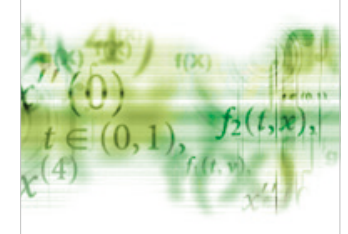

International Journal of

Differential Equations

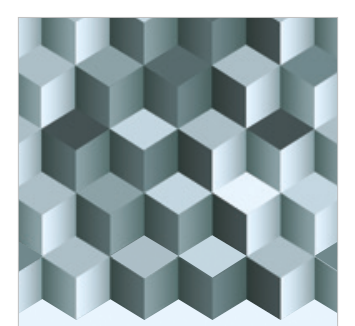

Journal of

Function Spaces
The Scientific

World Journal

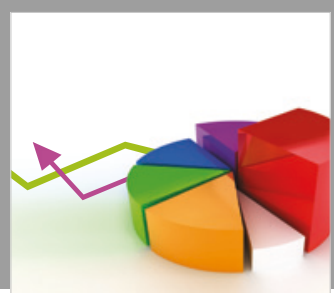

Journal of

Probability and Statistics
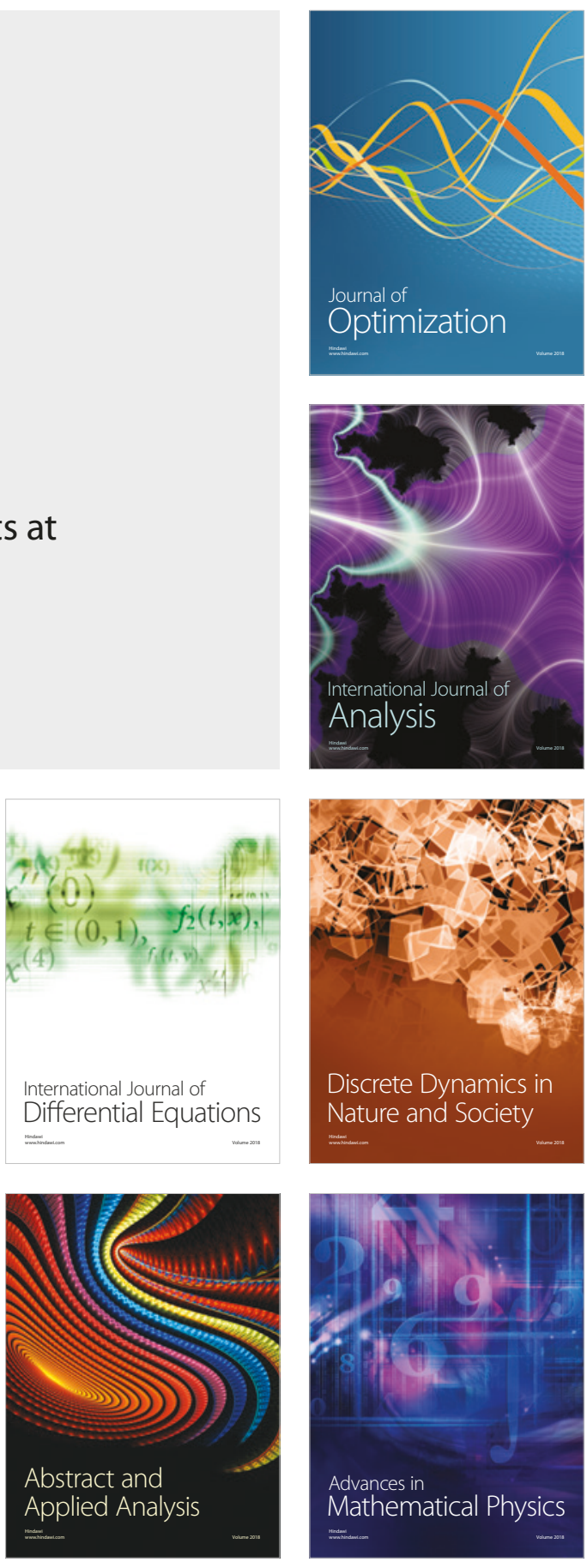